\title{
Added sugars and sugar-sweetened beverage consumption, dietary carbohydrate index and depression risk in the Seguimiento Universidad de Navarra (SUN) Project
}

\author{
Almudena Sanchez-Villegas ${ }^{1,2}$, Itziar Zazpe ${ }^{3}$, Susana Santiago ${ }^{3}$, Aurora Perez-Cornago ${ }^{4}$, \\ Miguel A. Martinez-Gonzalez ${ }^{2,5,6}$ and Francisca Lahortiga-Ramos ${ }^{7 *}$ \\ ${ }^{1}$ Nutrition Research Group, Research Institute of Biomedical and Health Sciences, University of Las Palmas de Gran Canaria, \\ Dr. Pasteur s/n, Trasera Hospital Insular, CP 35016, Las Palmas de Gran Canaria, Spain \\ ${ }^{2}$ Ciber de Fisiopatología de la Obesidad y Nutrición (CIBER OBN), Instituto de Salud Carlos III, 28029 Madrid, Spain \\ ${ }^{3}$ Department of Nutrition and Food Sciences and Physiology, University of Navarra, Irunlarrea s/n, 31008 Pamplona, Spain \\ ${ }^{4}$ Cancer Epidemiology Unit, Nuffield Department of Population Health, University of Oxford, Oxford OX3 7LF, UK \\ ${ }^{5}$ Department of Preventive Medicine and Public Health, University of Navarra, C/ Irunlarrea, $n^{\circ} 1$, CP 31008, Pamplona, Spain \\ ${ }^{6}$ Department of Nutrition, Harvard TH Chan School of Public Health, Boston, MA 02115, USA \\ ${ }^{7}$ Department of Psychiatry and Medical Psychology, University Clinic of Navarra, Pío XII 36, 31008 Pamplona, Navarra, \\ Spain
}

(Submitted 2 June 2017 - Final revision received 27 October 2017 - Accepted 2 November 2017-First published online 22 December 2017)

\section{Abstract}

The association between added sugars or sugar-sweetened beverage consumption and the risk of depression, as well as the role of carbohydrate quality in depression risk, remains unclear. Among 15546 Spanish university graduates from the Seguimiento Universidad de Navarra (SUN) prospective cohort study, diet was assessed with a validated 136-item semi-quantitative FFQ at baseline and at 10-year follow-up. Cumulative average consumption of added sugars, sweetened drinks and an overall carbohydrate quality index (CQI) were calculated. A better CQI was associated with higher whole-grain consumption and fibre intake and lower glycaemic index and consumption of solid (instead of liquid) carbohydrates. Clinical diagnoses of depression during follow-up were classified as incident cases. Multivariable timedependent Cox regression models were used to estimate hazard ratios (HR) of depression according to consumption of added sugars, sweetened drinks and CQI. We observed 769 incident cases of depression. Participants in the highest quartile of added sugars consumption showed a significant increment in the risk of depression ( $\mathrm{HR}=1 \cdot 35 ; 95 \% \mathrm{CI} 1 \cdot 09,1 \cdot 67, P=0 \cdot 034)$, whereas those in the highest quartile of CQI (upper quartile of the CQI) showed a relative risk reduction of $30 \%$ compared with those in the lowest quartile of the CQI (HR $=0 \cdot 70 ; 95 \%$ CI $0 \cdot 56,0 \cdot 88)$. No significant association between sugar-sweetened beverage consumption and depression risk was found. Higher added sugars and lower quality of carbohydrate consumption were associated with depression risk in the SUN Cohort. Further studies are necessary to confirm the reported results.

Key words: Added sugars: Sweetened beverages: Carbohydrate quality: Glycaemic index: Depression risk

Depression is one of the leading global causes of disabilityadjusted life years and the main cause in middle- and highincome countries $^{(1,2)}$. In the past years, several epidemiological studies have analysed the role of dietary patterns, foods, food groups and nutrients in the prevention of depression. Although evidence is still scarce, this is an emerging and promising field of research within nutritional epidemiology ${ }^{(3)}$.

Consumption of processed and sugary foods and beverages has exponentially increased worldwide, being in part responsible for the obesity and childhood obesity epidemic, and other non-communicable diseases such as type 2 diabetes mellitus and $\mathrm{CHD}^{(4)}$. Over the past few years, several prospective studies have observed an association between metabolic disturbances and inflammation with depression risk. Obesity has been found to be associated with elevated cortisol secretion and higher hypothalamic-pituitary-adrenal (HPA) axis reactivity to psychological and physiological stress, which may lead to altered endocrine and stress responses ${ }^{(5)}$. Moreover, a bidirectional

Abbreviations: CQI, carbohydrate quality index; GI, glycaemic index; GL, glycaemic load; HR, hazard ratio; MDS, Mediterranean Diet Score; SUN, Seguimiento Universidad de Navarra.

* Corresponding author: F. Lahortiga-Ramos, email flahortiga@unav.es 
relationship between obesity and depression cannot be ruled out $^{(6)}$, as risk factors for obesity may be also associated with the development of depressive disorders. In this context, a link between diets rich in sugars - as well as sweetened beverages and depression has been hypothesised. Several cross-sectional studies have analysed the association between the consumption of these products and depressive symptoms or perceived stress. For example, depressive symptoms were positively associated with the consumption of high-energy sweets in the study by Jeffery et $a l .{ }^{(7)}$. Mikolajczyk et $a l^{(8)}$ also found a positive association between fast food, snacks and sweet consumption and depressive symptoms and perceived stress among female students. Moreover, a prospective analysis within the National Institutes of Health-American Association of Retired Persons (NIH-AARP) Diet and Health Study observed a positive association between soft and fruit drinks consumption and the risk of depression $^{(9)}$. Finally, the Whitehall II cohort study has recently reported a positive association between a very high intake of sugar from sweet food/beverage and the risk of common mental disorders in males, but not in females ${ }^{(10)}$

However, the type, amount and quality of carbohydrates and sugary products and their association with the risk of depression has not been sufficiently studied. Therefore, the aim of this study was to assess the association between carbohydrate quality (measured using a carbohydrate quality index (CQI)), added sugars and sweetened drinks consumption with the risk of depression in the Seguimiento Universidad de Navarra (SUN) prospective cohort study ( $40 \cdot 6 \%$ males; mean age 37.2 years) after a median follow-up of $10 \cdot 3$ years.

\section{Methods}

\section{Study population}

The SUN Project is a prospective, dynamic cohort study, initiated in December 1999 in Spain. The sample is composed of Spanish university graduates. Its methods have been previously described $^{(11)}$. Information is gathered biennially by mailed or by Web-based questionnaires. The overall retention in the cohort approaches $90 \%$.

Up to December 2016, 22564 participants had completed the baseline questionnaire of the SUN Project. Participants who were lost to follow-up, who had not completed at least one follow-up questionnaire, who were outside of predefined limits for baseline total energy intake $(<3347 \mathrm{~kJ} / \mathrm{d}(<800 \mathrm{kcal} / \mathrm{d})$ or more than $16736 \mathrm{~kJ} / \mathrm{d}(4000 \mathrm{kcal} / \mathrm{d})$ in men, and $<2092 \mathrm{~kJ} / \mathrm{d}(<500 \mathrm{kcal} / \mathrm{d})$ or more than $14644 \mathrm{~kJ} / \mathrm{d}(3500 \mathrm{kcal} / \mathrm{d})$ in women) and participants with a prevalence of diabetes at baseline were excluded from the analyses. We also excluded those participants who were users of antidepressant medication or had reported a present or previous history of clinical diagnosis of depression (lifetime depression) at baseline and those with a depression diagnosis in the first 2 years of follow-up. Moreover, participants who reported the use of antidepressants during the follow-up but without a physician diagnosis of depression and participants with diabetes diagnosis during the follow-up and before the 10-year dietary assessment were not considered in the final analysis. Finally, 15546 participants were included in the prospective analyses for the present study (Fig. 1).
The study was approved by the Institutional Review Board of the University of Navarra. Written informed consent was not requested from the participants. Voluntary completion of the first questionnaire was considered to imply informed consent.

\section{Exposure assessment}

Dietary intake was assessed at baseline (Q_0) and after 10 years of follow-up (Q_10) with a 136-item, validated, semiquantitative $\mathrm{FFQ}^{(12,13)}$.

Nutrient intakes were calculated as frequency multiplied by nutrient composition of specified portion size for each food item using an ad hoc computer program specifically developed for this aim. A trained dietitian updated the nutrient database using the latest available information included in food composition tables for Spain ${ }^{(14,15)}$. Several food items such as yogurts (except natural yogurt), milkshakes, ice-creams and other dairies, cookies, muffins, pastries, chocolates and other candies and commercial bakeries, canned fruits, jam, honey, sugar-sweetened carbonated drinks and juices were considered to assess added sugars consumption.

The FFQ also included a question regarding sweetened drink consumption in the previous year. We considered sugarsweetened carbonated colas and fruit-flavoured carbonated sugar soft drinks as sweetened drinks.

The consumption of added sugars and sugar-sweetened drinks was adjusted for total energy intake using the residual method $^{(16)}$ and quartiles were built.

The $\mathrm{CQI}^{(17)}$ was calculated considering the following four criteria: the glycaemic index (GI) (negatively weighted); total dietary fibre intake $(\mathrm{g} / \mathrm{d})$; the ratio of solid carbohydrates:total (solid+liquid) carbohydrates; and finally the ratio of carbohydrates from whole grains:carbohydrates from total grains (whole grains + refined grains + products prepared with refined flours). Liquid carbohydrates were calculated by summing up sugarsweetened beverage and fruit juice consumption (four items), whereas solid carbohydrates corresponded to the carbohydrate content of the rest of the food with any carbohydrate content.

With the exception for the ratio of carbohydrates from whole grains:carbohydrates from total grains that was categorised into tertiles, the rest of the items were grouped into five categories (quintiles). The total score potential range was then from 4 to 18 , with higher values meaning better quality of carbohydrates. This total score was categorised into quartiles for the analyses.

Moreover, we also calculated quartiles of intake of each of the four components of the score separately (fibre, GI, ratio of solid:total carbohydrates and whole-grain:total-grain carbohydrate ratio in this last case analysed as tertiles of intake).

Finally, added sugars, sugar-sweetened drinks and the CQI and its components were also treated as continuous variables. $z$ Scores were calculated for the seven exposure variables. Each $z$ score was calculated as the exposure variable value minus the mean value for the exposure variable divided by standard deviation of the exposure variable.

To minimise any effect of variation in diet, we calculated the average cumulative consumption of added sugars, sweetened drinks and the CQI and their individual items by using baseline values and updated exposures with dietary data collected after 10 years of follow-up. 
$n 22564$ participants responded to the baseline questionnaire (December 2016)

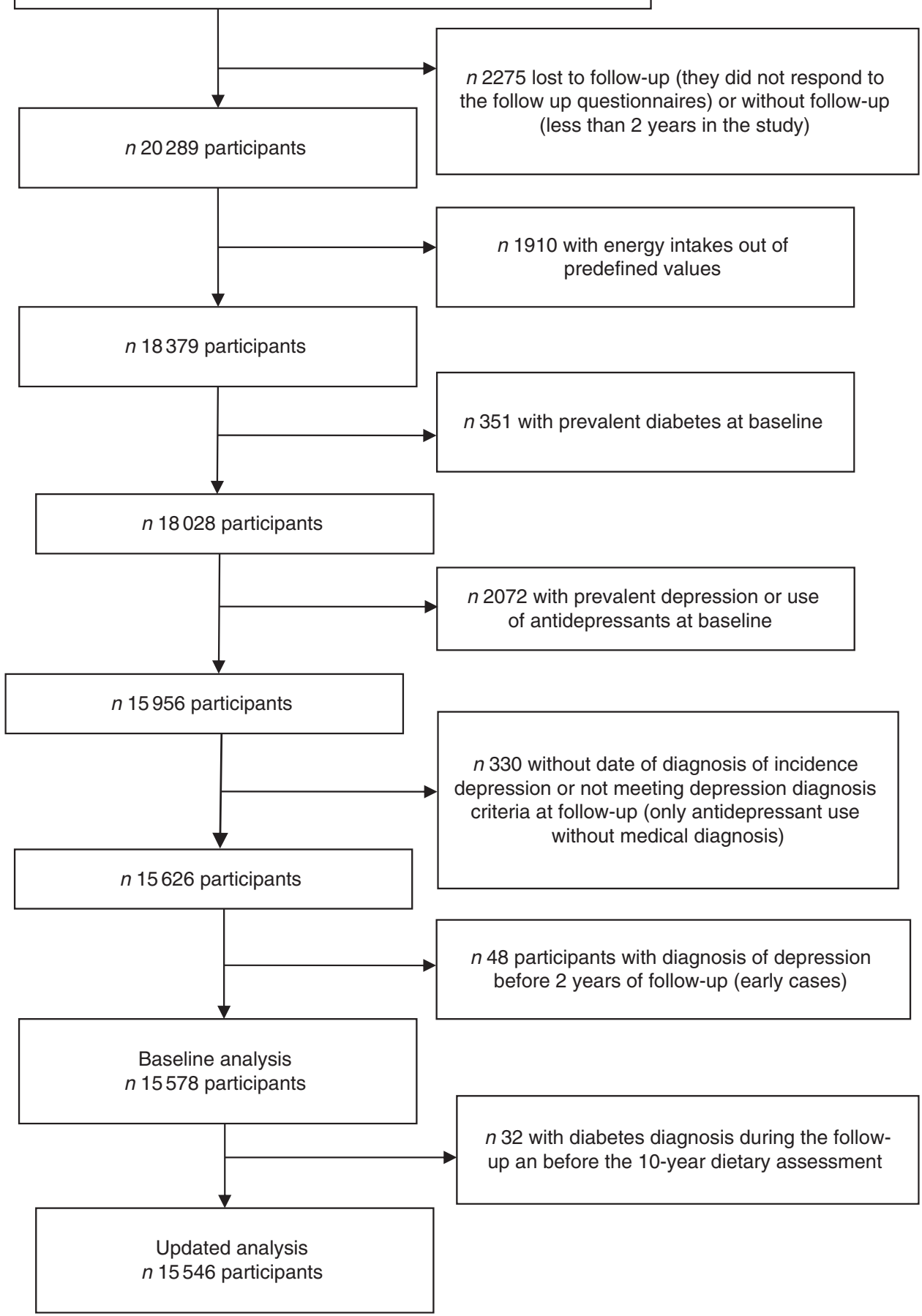

Fig. 1. Flow chart of participants. The Seguimiento Universidad de Navarra (SUN) Project.

\section{Outcome assessment}

Incident cases of depression were defined as participants who positively responded to the following question 'Have you ever been diagnosed of depression by a medical doctor?' in any of the biennial follow-up questionnaires from the fourth year of follow-up (Q_4-Q_16). We excluded those participants with a depression diagnosis before the first 2 years of follow-up from the analyses to avoid a possible reverse causality bias. It is possible that participants with subclinical depression at the beginning of our study might have changed their food habits precisely because of their mood disorder.

We did not consider antidepressant use during follow-up as a criterion to define incident depression (although the prescription 
of antidepressants for conditions other than depression is highly unusual in Spain). Therefore, we excluded from the analyses all the participants reporting the use of antidepressants during the follow-up who did not report any medical diagnosis of incident depression.

A self-reported physician-provided diagnosis of depression has demonstrated acceptable validity in a subsample of our cohort using the Structured Clinical Interview for DSM-IV as 'gold standard' applied by experienced psychiatrists blinded to the answers of the questionnaires ${ }^{(18)}$. The percentage of confirmed depression was $74.2 \%$ (95\% CI $63.3,85 \cdot 1)$. The percentage of confirmed non-depression was $81 \cdot 1 \%$ (95\% CI 69.1, 92.9).

\section{Other variables assessment}

Information about socio-demographic (e.g. sex, age, marital status and employment status) and lifestyle-related variables (e.g. smoking status, physical activity, special diets) were obtained from the Q_0. Smoking status was updated after eight years of follow-up (Q_8).

Physical activity was assessed using a validated physical activity questionnaire with data about seventeen activities ${ }^{(19)}$. Leisure-time activities were computed by assigning a metabolic equivalent score to each activity, multiplied by the time spent for each activity and summing up all activities.

BMI was calculated as weight $(\mathrm{kg})$ divided by the square of height $\left(\mathrm{m}^{2}\right)$ using data collected at baseline and after 10 years of follow-up (Q_10)

The prevalence and history of CVD, cancer, obesity, dyslipidaemia, hypertension and type 2 diabetes mellitus was ascertained at baseline and updated until the end of follow-up or until depression diagnosis was reported. CVD included myocardial infarction, stroke, atrial fibrillation, paroxysmal tachycardia, coronary artery bypass grafting or other re-vascularisation procedures, heart failure, aortic aneurism, pulmonary embolism or peripheral venous thrombosis. All the diagnoses were based on participants' selfreporting. The validity of self-reported obesity, dyslipidaemia and hypertension diagnoses has been assessed in different subsamples of the cohort ${ }^{(20-22)}$. Self-reported cardiovascular events, cancer and diabetes have been confirmed by medical record review.

Participants with prevalent diabetes mellitus at baseline and with a diabetes diagnosis during the follow-up and before the 10-year dietary assessment were excluded from the main analysis but they were included in a subsequent sensitivity analysis.

Energy intake, percentage of energy from fats, proteins and carbohydrates, consumption of fast food and commercial baked goods, and the adherence to the Mediterranean diet (according to the Mediterranean Diet Score (MDS) proposed by Trichopoulou ${ }^{(23)}$ ) were also calculated at baseline and after 10 years of follow-up through the FFQ.

\section{Statistical methods}

Pearson's correlation coefficients (correlation matrix) between each carbohydrate index components combination were calculated as it is possible that some of the components may be highly correlated.

For each participant, we computed person-years of follow-up from the date of returning the Q_0 to the date of depression diagnosis or the date of returning the last follow-up questionnaire, whichever came first.

Time-dependent Cox proportional-hazards regression models were fitted to assess the relationship between the energyadjusted added sugars and sweetened drink consumption and the CQI and the items used to its construction and the incidence of depression during follow-up. Hazard ratios (HR) and their 95\% CI were calculated considering the lowest quartile as the reference category. To control for potential confounding factors, we conducted both minimally adjusted models adjusting only for sex and age, and multivariable-adjusted models adjusting for sex, BMI ( $\mathrm{kg} / \mathrm{m}^{2}$, continuous), smoking (non-smoker, ex-smoker, current smoker, missing), physical activity during leisure time (METs/h-w), total energy intake $(\mathrm{kJ} / \mathrm{d}(\mathrm{kcal} / \mathrm{d})$, continuous), adherence to the MDS (0-9), continuous), the presence of several diseases (CVD, hypertension and dyslipidaemia) and year of recruitment (five categories). Age (analysed as deciles of age) was introduced as the underlying time variable and also as a stratifying factor.

To increase accuracy, energy intake and BMI were updated with the information obtained after 10 years of follow-up and smoking with information obtained after 8 years of follow-up. The prevalence of diseases (CVD, hypertension and dyslipidaemia) was updated using the information contained in any of the follow-up questionnaires. Participants who reported a diagnosis of diabetes after recruitment and before the 10-year assessment of dietary habits ( $n$ 32) were also excluded.

Other confounding factors such as the percentage of energy from fats or proteins in the diet, the consumption of fast food or commercial baked products, the use of special diets, marital status, employment status or prevalence of cancer were also explored but not included in the final models because their inclusion in the regression models did not substantially change the reported associations.

Tests of linear trend across increasing quartiles of exposures were conducted by assigning the medians to each quartile and treating them as continuous variables.

Time-dependent multivariable-adjusted Cox proportionalhazards regression models were also fitted to assess the association between each exposure $z$ score and depression risk.

To test for interaction by the prevalence of obesity or diabetes and the adherence to the MDS, we used likelihood ratio tests to compare models with and without the interaction product-term.

Several sensitivity analyses were conducted. We repeated all of the analyses including participants with diabetes at baseline. In addition, for the CQI analysis, we recalculated the index eliminating the ratio of carbohydrates from whole grains to carbohydrates from total grains (CQI-2) because of the low variability observed among the participants regarding wholegrains consumption, which is very unusual in Spain.

Finally, the potential non-parametrical non-linear association between the cumulative average CQI and CQI-2 and incident depression was calculated with restricted cubic splines ${ }^{(24)}$. Tests for non-linearity used the likelihood ratio test, comparing the model with only the linear term to the model with the linear and the cubic spline terms. We did not perform any kind of truncation of the data. The knots placements were 7, 11 and 14 for 
CQI and 6, 9 and 12 for CQI-2. The results were adjusted for the same potential confounding factors as the main Cox regression analysis.

All $P$ values were two-tailed, and $P<0.05$ was considered significant. Statistical analysis was performed using Stata version 12.0 (StataCorp).

\section{Results}

We recorded 769 incident cases of depression among 152931 person-years of follow-up.

Table 1 shows the distribution of socio-demographic and lifestyle characteristics of the sample at baseline according to quartiles of consumption of added sugars and sweetened drinks and the CQI

Participants with higher consumption of added sugars and sweetened drinks (fourth quartile) were more likely to be younger, male and showed a lower prevalence of CVD and cancer.

Participants in the highest quartile of the CQI were more likely to be women, older and physically more active, to use special diets and showed a higher prevalence of CVD, hypertension or dyslipidaemia. Moreover, they consumed less alcohol and more energy than participants in the lowest quartile.

The distribution of the incidence of depression according to the main baseline characteristics of the participants is shown in the online Supplementary Table S1. Incidence of depression was higher among females, unemployed subjects, current smokers and among those participants with low adherence to the MDS.

The association between the cumulative average consumption of energy-adjusted added sugars, sweetened drinks and the CQI and depression risk is shown in Table 2. The highest consumption of added sugars was associated with a higher risk of developing depression during the follow-up. In fact, participants in the upper quartiles of added sugars consumption showed a significant increment in the risk of depression (HR for the third quartile in the multivariable-adjusted model $=1.29$; 95\% CI 1.04,1.59; HR for the highest quartile $=1 \cdot 35 ; 95 \%$ CI 1.09, 1.67). In addition, a significant dose-response relationship was found $(P=0.034)$. However, when the results were adjusted for protein intake $(\% \mathrm{E})$, the association was attenuated and no longer significant for the highest intake. The HR and 95\% CI for the second, third and fourth quartiles were 1.20 (95\% CI $0.97,1.49), 1.31$ (95\% CI 1.06, 1.49) and 1.19 (95\% CI 0.96, $1 \cdot 47)$, respectively.

In the analysis of the CQI, an inverse relationship was observed when average cumulative dietary consumption was analysed. Participants with the highest quality in carbohydrate intake showed a relative risk reduction of $30 \%$ as compared with those with the lowest CQI (HR in multivariable-adjusted model $=0.70 ; 95 \%$ CI $0 \cdot 55,0 \cdot 88$ ).

The relationship between the baseline consumption of energyadjusted added sugars, sugar-sweetened drinks and the CQI and depression risk is shown in the Supplementary Table S2. We did not find any significant association using as exposure the baseline assessments. The HR for the highest $v$. the lowest quartile of baseline added sugars consumption and depression risk was 1.22 (95\% CI 0.98, 1.50).
None of the four singular criteria considered to build the CQI was significantly associated with depression risk, but their sum was. Although significant, the magnitude of the correlation coefficients between the different components of the CQI was low $(<0.30$ for all the possible comparisons with the exception of the GI and the ratio of solid:total carbohydrates, which showed a medium correlation) (online Supplementary Table S3). Thus, it is unlikely that collinearity could explain the reported results.

We did not find any significant effect modification (inter action) by the prevalence of obesity or diabetes in the beginning of the study, nor for the adherence to the MDS.

Several sensitivity analyses are shown in Table 3. The results did not change after including participants with diabetes at baseline. On the other hand, when the CQI was re-built eliminating the item ratio of carbohydrates from whole grains:carbohydrates from total grains' the association was strengthened (HR for the highest $v$. the lowest quartile $=0.62 ; 95 \%$ CI $0 \cdot 48,0.80$ ).

Finally, to account for non-linear associations, we used restricted cubic spline analysis. We found a suggestion of U-shaped associations (Fig. 2), indicating that moderate adherence to this index of carbohydrate quality should be responsible for a reduction in the risk of depression $(P<0 \cdot 001)$.

\section{Discussion}

In this analysis within the SUN cohort, high added sugars and lower quality of carbohydrate consumption was associated with an increased risk of depression. We did not find a significant association for sugar-sweetened beverage consumption in relation to depression risk.

The number of epidemiological studies that have analysed the role of added sugars, sugary foods and sweetened beverages in depression development is scarce. Moreover, most of them have a cross-sectional design and reverse causality cannot be excluded ${ }^{(7,8)}$. In other cases, although the results are based on analytical studies, these studies analysed overall dietary patterns ${ }^{(25)}$. In a recent case-control study, the highest adherence to an unhealthy dietary pattern that included sweets, soft drinks and industrial fruit juices was associated also with an increased risk of depression $(\mathrm{OR}=3.62 ; 95 \% \mathrm{CI} 1.59,8 \cdot 22)^{(26)}$. The Whitehall II prospective cohort also showed that a food pattern characterised by a high consumption of sweetened desserts, but also by fried food, processed meat, refined grains and high-fat dairy products, was associated with higher scores on the Center for Epidemiologic Studies-Depression scale ${ }^{(27)}$. In the same cohort, when sugar from sweet food/beverage was specifically analysed, the authors reported a positive association with common mental disorders but only among males ${ }^{(10)}$.

In a previous analysis of our cohort, the consumption of commercial bakery products was also related with a higher risk of depression ${ }^{(28)}$. We observed that participants who consumed more commercial bakery products had a $40 \%$ higher risk of depression compared with participants who did not consume these products or who had a very low intake. It might be hypothesised that the high content of pro-inflammatory transfatty acids in commercial bakery products may increase the risk of depression. In other analysis within the SUN cohort, transfatty acid intake was positively associated with the risk of 
Nes British Journal of Nutrition

Table 1. Baseline characteristics according to quartiles (Q) intake of added sugar and sweetened drinks and Carbohydrate Quality Index (CQI) at baseline (Mean values and standard deviations)

\begin{tabular}{|c|c|c|c|c|c|c|c|c|c|c|c|c|c|c|c|c|c|c|}
\hline & \multicolumn{8}{|c|}{ Energy-adjusted added sugars (g/d) } & \multirow[b]{3}{*}{$P^{\star}$} & \multicolumn{8}{|c|}{ Energy-adjusted sweetened drinks (g/d) } & \multirow[b]{3}{*}{$P^{*}$} \\
\hline & \multicolumn{2}{|c|}{ Q1 } & \multicolumn{2}{|c|}{ Q2 } & \multicolumn{2}{|c|}{ Q3 } & \multicolumn{2}{|c|}{ Q4 } & & \multicolumn{2}{|c|}{ Q1 } & \multicolumn{2}{|c|}{ Q2 } & \multicolumn{2}{|c|}{ Q3 } & \multicolumn{2}{|c|}{ Q4 } & \\
\hline & Mean & SD & Mean & SD & Mean & SD & Mean & SD & & Mean & SD & Mean & SD & Mean & SD & Mean & SD & \\
\hline Age (years) & $40 \cdot 0$ & $12 \cdot 7$ & 37.3 & $12 \cdot 1$ & $36 \cdot 1$ & $11 \cdot 8$ & 35.4 & 11.3 & $<0.001$ & 38.6 & 11.9 & 39.0 & 12.7 & 37.8 & 12.6 & 33.4 & $10 \cdot 3$ & $<0.001$ \\
\hline Male (\%) & $37 \cdot \varepsilon$ & & 37 & 7.8 & 41. & & 45 & & $<0.001$ & 11 & & 39 & $9 \cdot 1$ & 59 & 9.4 & 52 & & $<0.001$ \\
\hline Unemployed (\%) & 3.6 & & 4 & 2 & 4.5 & & 3.9 & & 0.237 & 4.1 & & 3. & & 4. & & 4.4 & & 0.233 \\
\hline Married (\%) & $57 \cdot$ & & 50 & 3 & 47. & & 47 & & $<0.001$ & 54 & & 55 & & 52 & & 40 & & $<0.001$ \\
\hline Special diets (\%) & 8.9 & & 6 & 9 & 6.5 & & 6.4 & & $<0.001$ & 7.8 & & 9 & & 6 & & 4.8 & & $<0.001$ \\
\hline Smoking status (\%) & & & & & & & & & $<0.001$ & & & & & & & & & $<0.001$ \\
\hline Ex-smoker & $28 \cdot \varepsilon$ & & 24 & 4.3 & 23. & & 21. & & & 27 & & 28 & & 24 & & 18 & & \\
\hline Current smoker & 25 & & 23 & 3.4 & 23 . & & 25 & & & 21 & & 21 & & 23 & & 31. & & \\
\hline \multicolumn{19}{|l|}{ Prev. diseases (\%) } \\
\hline $\begin{array}{l}\text { CVD } \\
\text { Cos (1) }\end{array}$ & 3.4 & & 2 & .9 & 2.4 & & 2.4 & & 0.018 & 2.6 & & 3. & 3.3 & 3. & & 1.8 & & $<0.001$ \\
\hline Cancer & 3.7 & & 3 & 3 & 3.6 & & 2.8 & & 0.097 & 4.5 & & 3 & 3.6 & 2. & & 2.0 & & $<0.001$ \\
\hline Hypertension & 8.5 & & 6 & 2 & 6. & & 5.6 & & $<0.001$ & 4.8 & & 7 & .7 & 8. & & 5.0 & & $<0.001$ \\
\hline Dyslipidaemia & 19 & & 16 & 5.8 & 17 & & $17 \cdot($ & & 0.056 & 15 & & 19 & & 20 & & 15 & & $<0.001$ \\
\hline $\mathrm{BMI}\left(\mathrm{kg} / \mathrm{m}^{2}\right)$ & 23.8 & 3.7 & 23.4 & 3.4 & 23.3 & 3.3 & 23.4 & 3.4 & $<0.001$ & 22.6 & 3.0 & 23.5 & 3.6 & 24.1 & 3.5 & 23.7 & 3.5 & $<0.001$ \\
\hline Physical activity (METs-h/week) & $22 \cdot 2$ & $23 \cdot 3$ & 22.1 & 22.4 & 22.0 & 23.0 & 22.5 & 23.5 & 0.7917 & 22.3 & 22.7 & 22.1 & 22.9 & 22.5 & 23.4 & 21.8 & 23.2 & 0.5746 \\
\hline Energy intake $(\mathrm{kJ} / \mathrm{d})$ & 10330 & 2443 & 9372 & 2540 & 9414 & 2548 & 10640 & 2590 & $<0.001$ & 10837 & 2239 & 9381 & 2439 & 8828 & 2443 & 10343 & 2653 & $<0.001$ \\
\hline Energy intake (kcal/d) & 2469 & 584 & 2240 & 607 & 2250 & 609 & 2453 & 619 & & 2590 & 535 & 2242 & 583 & 2110 & 584 & 2472 & 634 & $<0.001$ \\
\hline Alcohol intake $(\mathrm{g} / \mathrm{d})$ & 8.5 & 13.2 & 6.4 & 8.9 & 6.0 & 8.4 & 5.8 & 8.4 & $<0.001$ & 5.7 & 10.0 & 6.6 & 10.7 & 6.7 & 9.2 & 7.7 & 10.0 & $<0.001$ \\
\hline Carbohydrate intake (\%E) & $41 \cdot 3$ & 8.0 & $42 \cdot 3$ & 7.2 & 43.5 & 6.7 & 46.0 & 6.5 & $<0.001$ & 44.1 & 7.5 & 43.2 & 7.6 & 42.5 & 7.2 & 43.2 & 6.9 & $<0.001$ \\
\hline Protein intake $(\% \mathrm{E})$ & 18.7 & 3.4 & 18.8 & 3.2 & 18.0 & 3.0 & 17.0 & 3.1 & $<0.001$ & 18.0 & 3.2 & 18.5 & 3.5 & 18.5 & 3.3 & 17.4 & 2.9 & $<0.001$ \\
\hline Fat intake (\%E) & 37.5 & $7 \cdot 2$ & 36.9 & 6.5 & 36.6 & 6.1 & $35 \cdot 3$ & 6.0 & $<0.001$ & $36 \cdot 3$ & 6.8 & $36 \cdot 2$ & 6.8 & 36.7 & 6.4 & 37.1 & $6 \cdot 1$ & $<0.001$ \\
\hline $\operatorname{MDS}(0-9)$ & 4.9 & 1.7 & 4.3 & 1.7 & 4.0 & 1.7 & 3.7 & 1.6 & $<0.001$ & 4.7 & 1.7 & 4.4 & 1.7 & 4.0 & 1.7 & 3.9 & 1.7 & $<0.001$ \\
\hline Energy-adj. fast-food consumption & 21.6 & 22.8 & 22.9 & $18 \cdot 7$ & $22 \cdot 9$ & 18.5 & $22 \cdot 3$ & 19.6 & 0.0115 & $17 \cdot 2$ & $19 \cdot 7$ & 20.0 & 17.1 & $24 \cdot 2$ & $18 \cdot 4$ & $28 \cdot 2$ & $22 \cdot 5$ & $<0.001$ \\
\hline Energy-adj. commercial baked goods consumption & 10.2 & 15.7 & 13.5 & 15.7 & 15.6 & 18.3 & 18.5 & $24 \cdot 1$ & $<0.001$ & 10.3 & 19.7 & $13 \cdot 1$ & $16 \cdot 4$ & $16 \cdot 2$ & $17 \cdot 4$ & $18 \cdot 2$ & $21 \cdot 1$ & $<0.001$ \\
\hline & & & & & CQI & & & & & & & & & & & & & \\
\hline & Q1 & & Q & 22 & Q3 & & Q4 & & & & & & & & & & & \\
\hline & Mean & SD & Mean & SD & Mean & SD & Mean & SD & $P^{\star}$ & & & & & & & & & \\
\hline Age (years) & 35.5 & 11.6 & 37.1 & 11.8 & 38.0 & $12 \cdot 3$ & 39.7 & $12 \cdot 8$ & $<0.001$ & & & & & & & & & \\
\hline Male $(\%)$ & $47 \cdot \varepsilon$ & & 40 & 3 & 35 & & 32 & & $<0.001$ & & & & & & & & & \\
\hline Unemployed (\%) & 4.7 & & 3 & 8 & 3.8 & & 3.5 & & 0.028 & & & & & & & & & \\
\hline Married (\%) & 48 & & 50 & 5.8 & 53 . & & 51. & & $<0.001$ & & & & & & & & & \\
\hline Special diets (\%) & 4.4 & & 6 & 9 & 8.4 & & $11 \cdot \varepsilon$ & & $<0.001$ & & & & & & & & & \\
\hline Smoking status (\%) & & & & & & & & & $<0.001$ & & & & & & & & & \\
\hline Ex-smoker & 21.8 & & 24 & $4 \cdot 1$ & 25 & & $29 \cdot($ & & & & & & & & & & & \\
\hline Current smoker & 30. & & 24 & 4.2 & 21 & & $17 \cdot \varepsilon$ & & & & & & & & & & & \\
\hline Prev. diseases (\%) & & & & & & & & & & & & & & & & & & \\
\hline CVD & 2.4 & & 2 & .8 & 2.9 & & 3.5 & & 0.062 & & & & & & & & & \\
\hline Cancer & 3.1 & & 2 & 6 & 3.6 & & 4.0 & & 0.007 & & & & & & & & & \\
\hline Hypertension & 5.9 & & 6 & 1 & 7.6 & & 7.4 & & 0.004 & & & & & & & & & \\
\hline Dyslipidaemia & 17 & & 17 & 7.8 & 17 & & 18 & & 0.464 & & & & & & & & & \\
\hline $\operatorname{BMI}\left(\mathrm{kg} / \mathrm{m}^{2}\right)$ & 23.6 & 3.5 & 23.4 & 3.4 & 23.5 & 3.4 & $23 \cdot 3$ & 3.4 & 0.0273 & & & & & & & & & \\
\hline Physical activity (METs-h/week) & $20 \cdot 1$ & $20 \cdot 8$ & $21 \cdot 1$ & 20.9 & $24 \cdot 3$ & 25.5 & 25.9 & $26 \cdot 3$ & $<0.001$ & & & & & & & & & \\
\hline Energy intake $(\mathrm{kJ} / \mathrm{d})$ & 9259 & 2502 & 9916 & 2565 & 10272 & 2577 & 10410 & 2469 & $<0.001$ & & & & & & & & & \\
\hline Energy intake $(\mathrm{kcal} / \mathrm{d})$ & 2213 & 598 & 2370 & 613 & 2455 & 616 & 2488 & 590 & $<0.001$ & & & & & & & & & \\
\hline Alcohol intake $(\mathrm{g} / \mathrm{d})$ & 7.5 & 11.5 & 6.6 & 9.6 & 6.2 & 9.1 & 5.5 & 7.8 & $<0.001$ & & & & & & & & & \\
\hline Carbohydrate intake (\%E) & 42.4 & $7 \cdot 2$ & 43.0 & 7.1 & 43.9 & 7.5 & 44.6 & 7.4 & $<0.001$ & & & & & & & & & \\
\hline Protein intake $(\% \mathrm{E})$ & 17.7 & 3.3 & $18 \cdot 1$ & 3.1 & 18.4 & 3.2 & 18.7 & 3.3 & $<0.001$ & & & & & & & & & \\
\hline Fat intake (\%E) & 37.4 & 6.5 & 36.9 & 6.3 & 35.9 & 6.6 & 35.2 & 6.5 & $<0.001$ & & & & & & & & & \\
\hline MDS $(0-9)$ & 3.4 & 1.5 & 4.2 & 1.6 & 4.8 & 1.6 & 5.4 & 1.5 & $<0.001$ & & & & & & & & & \\
\hline Energy-adj. fast-food consumption & $24 \cdot 3$ & $19 \cdot 0$ & 22.8 & 19.5 & $21 \cdot 1$ & 20.4 & 19.6 & 21.8 & $<0.001$ & & & & & & & & & \\
\hline Energy-adj. commercial baked goods consumption & 16.6 & $18 \cdot 7$ & $15 \cdot 3$ & 19.5 & $13 \cdot 2$ & 20.3 & $10 \cdot 3$ & 16.0 & $<0.001$ & & & & & & & & & \\
\hline
\end{tabular}


Table 2. Association between quartiles $(\mathrm{Q})$ of updated intake of added sugars, sweetened drinks, Carbohydrate Quality Index and depression (Hazard ratios (HR) and $95 \%$ confidence intervals)

\begin{tabular}{|c|c|c|c|c|c|c|c|}
\hline & \multirow[b]{2}{*}{ Median } & \multirow[b]{2}{*}{ Cases } & \multirow[b]{2}{*}{ Person-years } & \multicolumn{2}{|c|}{ Model $1^{*}$} & \multicolumn{2}{|c|}{ Model $2 \dagger$} \\
\hline & & & & HR & $95 \% \mathrm{Cl}$ & HR & $95 \% \mathrm{Cl}$ \\
\hline \multicolumn{8}{|c|}{ Energy-adjusted added sugars (g/d) } \\
\hline Q1 & 29.3 & 169 & 36978 & 1 & Ref. & 1 & Ref. \\
\hline Q2 & $45 \cdot 8$ & 185 & 38098 & 1.16 & $0.94,1.43$ & 1.15 & $0.93,1.43$ \\
\hline Q3 & $60 \cdot 0$ & 202 & 38593 & 1.29 & $1.04,1.59$ & 1.29 & $1.04,1.59$ \\
\hline Q4 & $83 \cdot 2$ & 213 & 39263 & 1.38 & $1 \cdot 12,1 \cdot 70$ & 1.35 & $1.09,1.67$ \\
\hline$P$ for trend & & & & \multicolumn{2}{|c|}{0.019} & \multicolumn{2}{|c|}{0.034} \\
\hline$z$ score & & & & $1 \cdot 10$ & $1 \cdot 02,1 \cdot 19$ & 1.09 & $1.01,1.18$ \\
\hline \multicolumn{8}{|c|}{ Energy-adjusted sweetened drinks (g/d) } \\
\hline Q1 & 1.3 & 213 & 37281 & 1 & Ref. & 1 & Ref. \\
\hline Q2 & $15 \cdot 5$ & 205 & 37853 & 1.15 & $0.95,1.40$ & 1.23 & $1.00,1.51$ \\
\hline Q3 & $30 \cdot 3$ & 163 & 38525 & 0.99 & $0.79,1.23$ & 1.04 & $0.83,1.32$ \\
\hline Q4 & $95 \cdot 7$ & 188 & 39272 & $1 \cdot 14$ & $0.92,1.42$ & 1.12 & $0.90,1.41$ \\
\hline$P$ for trend & & & & \multicolumn{2}{|c|}{0.022} & \multicolumn{2}{|c|}{0.090} \\
\hline z score & & & & 1.03 & $0.95,1.10$ & 1.00 & $0.93,1.09$ \\
\hline \multicolumn{8}{|c|}{ Carbohydrate Quality Index (4-18) } \\
\hline Q1 & 8 & 262 & 54993 & 1 & Ref. & 1 & Ref. \\
\hline Q2 & 10 & 208 & 40419 & 1.17 & $0.97,1.41$ & 1.15 & $0.87,1.27$ \\
\hline Q3 & 12 & 177 & 34216 & 0.80 & $0.66,0.97$ & 0.81 & $0.66,1.00$ \\
\hline Q4 & 15 & 121 & 23301 & 0.66 & $0.53,0.83$ & 0.70 & $0.56,0.88$ \\
\hline$P$ for trend & & & & \multicolumn{2}{|c|}{0.063} & \multicolumn{2}{|c|}{0.401} \\
\hline$z$ score & & & & 0.96 & $0.89,1.04$ & 1.01 & $0.92,1.10$ \\
\hline \multicolumn{8}{|c|}{ Glycaemic index } \\
\hline Q4 & $57 \cdot 1$ & 184 & 39512 & 1 & Ref. & 1 & Ref. \\
\hline Q3 & $53 \cdot 6$ & 193 & 38383 & 1.06 & $0.86,1.30$ & 1.07 & $0.88,1.32$ \\
\hline Q2 & $50 \cdot 9$ & 198 & 37891 & 1.02 & $0.83,1.25$ & 1.07 & $0.87,1.31$ \\
\hline Q1 & $47 \cdot 2$ & 193 & 37143 & 1.02 & $0.83,1.26$ & 1.13 & $0.91,1.40$ \\
\hline$P$ for trend & & & & \multicolumn{2}{|c|}{0.608} & \multicolumn{2}{|c|}{0.153} \\
\hline$z$ score & & & & 0.98 & $0.91,1.05$ & 0.96 & $0.89,1.04$ \\
\hline \multicolumn{8}{|l|}{ Fibre intake } \\
\hline Q1 & $15 \cdot 9$ & 200 & 38625 & 1 & Ref. & 1 & Ref. \\
\hline Q2 & $22 \cdot 7$ & 192 & 38622 & 0.95 & $0.78,1.15$ & 0.92 & $0.75,1.14$ \\
\hline Q3 & $29 \cdot 2$ & 184 & 38310 & 0.83 & $0.67,1.02$ & 0.79 & $0.62,1.00$ \\
\hline Q4 & $40 \cdot 6$ & 192 & 37372 & 0.86 & $0.70,1.05$ & 0.79 & $0.60,1.05$ \\
\hline$P$ for trend & & & & \multicolumn{2}{|c|}{0.242} & \multicolumn{2}{|c|}{0.291} \\
\hline$z$ score & & & & 0.97 & $0.90,1.04$ & 0.99 & $0.90,1 \cdot 10$ \\
\hline Ratio of solid:t & ohydrate & & & & & & \\
\hline Q1 & 0.79 & 212 & 38206 & 1 & Ref. & 1 & Ref. \\
\hline Q2 & 0.86 & 173 & 38367 & 0.83 & $0.68,1.02$ & 0.85 & $0.69,1.04$ \\
\hline Q3 & 0.90 & 212 & 38410 & 0.99 & $0.82,1.20$ & 1.02 & $0.84,1.24$ \\
\hline Q4 & 0.95 & 172 & 37948 & 0.83 & $0.67,1.01$ & 0.86 & $0.70,1.06$ \\
\hline$P$ for trend & & & & & & & \\
\hline$z$ score & & & & 0.93 & $0.87,1.01$ & 0.95 & $0.87,1.03$ \\
\hline Ratio of whole & otal carbc & & & & & & \\
\hline $\mathrm{T} 1$ & & 491 & 99617 & 1 & Ref. & 1 & Ref. \\
\hline T2 & 0 & 20 & 4414 & 0.33 & $0.22,0.51$ & 0.34 & $0.22,0.51$ \\
\hline T3 & 0.01 & 258 & 48901 & 0.89 & $0.76,1.03$ & 0.96 & $0.82,1.12$ \\
\hline$P$ for trend & 0.15 & & & & & & \\
\hline$z$ score & & & & 1.05 & $0.98,1.13$ & 1.08 & $1.01,1.16$ \\
\hline
\end{tabular}

Ref., referent values; $\mathrm{T}$, tertiles.

* Model 1: cumulative average for each dietary factor intake (at baseline and after 10 years of follow-up). Adjusted for sex. The analyses were stratified by age groups.

† Model 2: cumulative average for each dietary factor intake (at baseline and after 10 years of follow-up). Adjusted for sex, smoking, BMl, physical activity, energy intake, adherence to the Mediterranean diet, prevalence of CVD, hypertension or dyslipidaemia and recruitment period. The analyses were stratified by age groups. Energy intake, BMI, smoking and prevalence of diseases were also updated. Participants with a diagnosis of diabetes during the follow-up and before the 10 -year assessment of diet were excluded ( $n$ 32).

depression $^{(29)}$. Nevertheless, the presence of sugars and other carbohydrates in bakery and pastries should also be taken into account.

Carbohydrate intake has been associated with improvement in mental performance and stress control through mechanisms modulating plasma concentrations of tryptophan and of large neutral amino acids (LNAA) ${ }^{(30)}$. The ability of dietary carbohydrates to modify the plasma amino acid pattern and to increase the uptake of circulating tryptophan into the brain depends on its ability to promote the secretion of insulin. Insulin has slight or no effect on plasma tryptophan, but it may decrease plasma levels of LNAA, which compete with tryptophan for passage across the blood-brain barrier. This decrease allows more tryptophan to enter the brain after dietary carbohydrate intake. Several experimental studies have suggested that a variation of $50 \%$ in the plasma tryptophan:LNAA ratio is sufficient to change significantly the release of serotonin, as tryptophan is the most important essential amino acid precursor of serotonin ${ }^{(31)}$. 

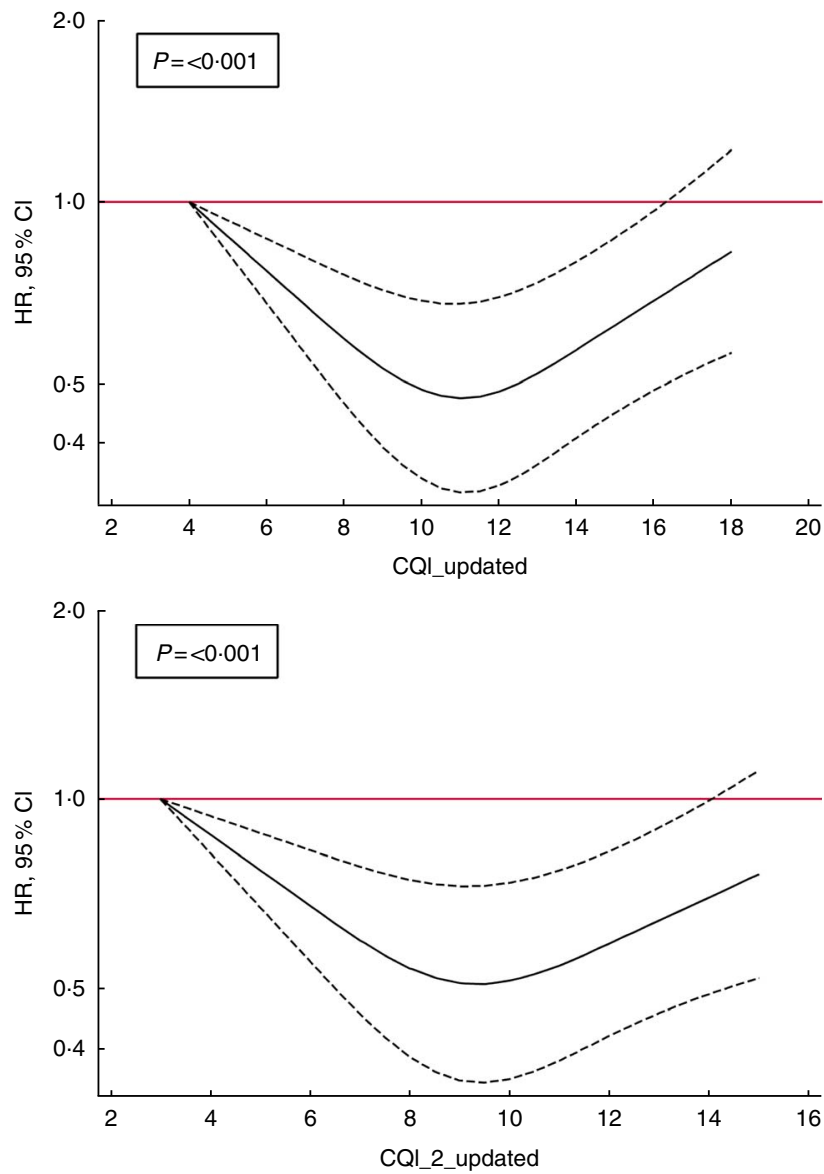

Fig. 2. Spline regression models of the hazard ratio (HR) of depression according to Carbohydrate Quality Index (CQI) and CQI 2 without the ratio of whole grains/total carbohydrate $(\mathrm{CH})$ (repeated measures) $(-------, 95 \% \mathrm{Cl})$. Likelihood ratio test was used to test for non-linearity. Adjusted for sex, smoking, BMI, physical activity, energy intake, adherence to the Mediterranean diet, prevalence of CVD, hypertension or dyslipidaemia and recruitment period. The analyses were stratified by age groups. Energy intake, BMI, smoking and prevalence of diseases were also updated. Participants with a diagnosis of diabetes during the follow-up and before the 10-year assessment of diet were excluded ( $n$ 32).

However, carbohydrate intake has not been associated with depression in the few small cross-sectional epidemiological studies that have analysed this hypothesis ${ }^{(32-34)}$. To our knowledge, there are no longitudinal studies that have previously analysed this association.

Among the different types of carbohydrates, overconsumption of fructose has raised as an important public health issue. Fructose-containing added sugars are a focus of attention for public health policies because several studies points out towards an increase in the risk of diabetes, metabolic syndrome, obesity or CVD associated with their use ${ }^{(35)}$, although this effect might be attributed to liquid sources such as sweetened beverages ${ }^{(4)}$. In fact, consumption of added sugars from liquid but not solid sources has been related with several metabolic disturbances such as impaired glucose homoeostasis and insulin resistance ${ }^{(36)}$.

On the other hand, few studies have assessed the association between fructose consumption and depression risk. Only some animal studies have been carried out in which fructose ingestion has been related with a stimulation of the HPA axis leading to 
elevations in glucocorticoids. In the study by Harrell et al., the rats fed a high-fructose diet during their periadolescence showed behavioural and hormonal responses with increased depressive-like behaviour and elevated basal corticosterone concentration in adulthood as compared with their chow-fed peers $^{(37)}$.

Other characteristics related to the quality of carbohydrates, such as the GI or the fibre content, might be important for disease development. To our knowledge, this is the first prospective study that has found a significant association between an index designed to assess the quality of carbohydrates in diet and the risk of depression. Although the individual components of the index were not associated with depression risk, the sum of the four of them, which represents an overall quality of carbohydrate intake, was associated with a lower risk of developing depressive disorders during the follow-up.

The beneficial role of fibre and whole-grain intake in the prevention of CVD and especially of diabetes has been extensively demonstrated for a large number of epidemiological studies $^{(38)}$. In addition, several systematic reviews have addressed the detrimental role of high GI and glycaemic load (GL) in cardio-metabolic conditions ${ }^{(39,40)}$. The GI is an indicator that measures how carbohydrate-containing foods raise blood glucose in comparison with a reference food, usually glucose or white bread ${ }^{(41)}$. The GL reflects both the quality and quantity of carbohydrate. Diets with low GI and GL are able to reduce the level of fasting insulin and also improve the lipid profile ${ }^{(42)}$. Moreover, the anti-inflammatory effect of these diets has been suggested $^{(43,44)}$. For example, in a recent analysis from a small cohort study, a higher intake of carbohydrates from higher-GI food sources and lower whole-grain consumption during puberty predicted greater IL-6 concentrations in young adulthood, although the authors did not find a significant association with fibre intake ${ }^{(44)}$.

Metabolic disturbances and inflammatory processes are present in both cardio-metabolic diseases and depressive disorders. Although diets with high GI might be associated with lower risk of depressive disorders because of their effect on insulin secretion, which facilitates the transport of tryptophan in the brain, the link between low-GI, fibre or whole-grain consumption and depression risk reduction seems plausible through their anti-inflammatory mechanisms. In a recent study published in 2016, Gopinath et al. ${ }^{(45)}$ found a positive association between dietary GI and depression prevalence. The same result was found by Haghighatdoost et al. ${ }^{(46)}$, although in this analysis the association was inverse for GL. More importantly, Breymeyer et $a l^{(47)}$ carried out a clinical trial with low/high-GL diets. Participants assigned to a high-GL diet showed higher depression symptoms, total mood disturbance and fatigue as compared with those assigned to a low-GL diet. These results had been observed in other clinical trials ${ }^{(48)}$, although not in all ${ }^{(33)}$

It is important to highlight that high-GI and high-GL diets may contain lower amounts of fibre and whole grains than low-GI and low-GL diets, which could explain the detrimental role of high GI in depression. In fact, some studies have found an inverse association between fibre intake and depression prevalence ${ }^{(45,49)}$. The hypothesised role of fibre intake in depression development might be related with gut-microbiota composition and activity, including some mechanisms linked with the gut-brain axis, as well as with immune, neural and metabolic pathways involved in depression pathogenesis ${ }^{(50)}$

Our analyses revealed a U-shaped relationship between CQI and depression, similar to that observed for the role of the adherence to healthy dietary patterns in depression in a previous analysis within the cohort ${ }^{(51)}$. In this sense, moderate carbohydrate quality as we observed for moderate adherence to diet quality patterns (but not the highest quality in carbohydrates, nor the highest diet patterns quality) showed the strongest inverse association with depression. It could be speculated that some psychological elements of neurotic or obsessive traits present in some participants classified in the highest category of quality (both for carbohydrates and healthy dietary patterns) may contribute to the observed plateau reached at moderate level of quality.

Strengths of this study deserve to be mentioned and include its prospective longitudinal design, the use of previously validated methods, the large sample size and the repeated measurements of the diet. Participants were highly educated, which increases the quality of self-reported information and reduces the potential for misclassification bias ${ }^{(52)}$. In addition, the restriction to a fairly homogeneous subgroup of participants with regard to educational level minimises the potential for residual confounding and is an excellent technique to improve the internal validity of our results. In fact, to maximise the validity of a cohort, it is recommended by methodologists to 'select study groups for homogeneity with respect to important confounders, for highly cooperative behaviours and for availability of accurate information, rather than attempt to be representative of a natural population ${ }^{(53)}$. We also excluded cases of depression diagnosed within the first 2 years of follow-up to try to avoid that some possible results would be explained by a reverse causation bias owing to subclinical cases of depression present at baseline.

Some possible limitations should also be mentioned. The SUN cohort is not a representative sample of the general population, which could limit the generalisability of our findings to other populations, and therefore generalisation of these results should be based on biological mechanisms rather than on statistical representativeness. Dietary intake and diagnosis of depression were self-reported; however, both methods have been previously validated in our cohort ${ }^{(12,13,18)}$. Although all the results were adjusted for a variety of major potential confounders, we cannot exclude the presence of some unknown or unmeasured factors that could partly explain the reported results. Some unmeasured dietary factors could partly explain the reported results. Moreover, participants' degree of loneliness or social network is not collected in our cohort. Recently, it has been suggested that social isolation would lead to increased level of sugar intake ${ }^{(54)}$.

The restriction of the sample to a homogeneous educated subgroup of participants could lead to little variability in the exposure that might have reduced the possibility of significant findings. Moreover, highly educated participants in this cohort might be more aware of the negative effects of high-sugar diets and other detrimental foods and, thus, social desirability bias might not be excluded. The presence of this non-differential 
misclassification bias would lead the associations towards the null. Finally, the observed non-linear associations could indicate that some findings could be explained, in part, by chance.

In conclusion, higher exposure to added sugars and poor quality of carbohydrates were associated with a higher risk of depression in this study. To date, most of the available studies that had analysed the role of total carbohydrates, sugars, GI or fibre in depression were prospectively the possible association between carbohydrate quantities, quality and types and depression risk. More studies similar to the SUN Cohort study with longitudinal design are needed to confirm the reported results.

\section{Acknowledgements}

The authors thank the participants and the members of the SUN project.

The SUN Project has received funding from the Spanish Government (grants PI10/02658, PI10/02293, PI13/00615, PI14/ 01798, RD06/0045, G03/140 and 87/2010), the Navarra Regional Government $(45 / 2011,27 / 2011)$ and the University of Navarra.

The authors' responsibilities were as follows: A. S.-V. and M. A. M.-G. designed the research, wrote the paper and had primary responsibility for the final content; A. S.-V. analysed and interpreted the data; I. Z., S. S., A. P.-C. and F. L.-R. revised the manuscript for important intellectual content; and all authors read and approved the final manuscript.

None of the authors has any conflicts of interest to declare.

\section{Supplementary material}

For supplementary material's referred to in this article, please visit https://doi.org/10.1017/S0007114517003361

\section{References}

1. GBD 2015 DALYs and HALE Collaborators (2016) Global, regional, and national disability-adjusted life-years (DALYs) for 315 diseases and injuries and healthy life expectancy (HALE), 1990-2015: a systematic analysis for the Global Burden of Disease Study 2015. Lancet 388, 1603-1658.

2. Global Burden of Disease Study 2013 Collaborators (2015) Global, regional, and national incidence, prevalence, and years lived with disability for 301 acute and chronic diseases and injuries in 188 countries, 1990-2013: a systematic analysis for the Global Burden of Disease Study 2013. Lancet 386, 743-800.

3. Sarris J, Logan AC, Akbaraly TN, et al. (2015) Nutritional medicine as mainstream in psychiatry. Lancet Psychiatry 2, 271-274.

4. Rippe JM \& Angelopoulos TJ (2016) Sugars, obesity, and cardiovascular disease: results from recent randomized control trials. Eur J Nutr 55, Suppl. 2, 45-53.

5. McInnis CM, Thoma MV, Gianferante D, et al. (2014) Measures of adiposity predict interleukin-6 responses to repeated psychosocial stress. Brain Behav Immun 42, 33-40.

6. Mannan M, Mamun A, Doi S, et al. (2016) Prospective associations between depression and obesity for adolescent males and females - a systematic review and meta-analysis of longitudinal studies. PLOS ONE 11, e0157240.

7. Jeffery RW, Linde JA, Simon GE, et al. (2009) Reported food choices in older women in relation to body mass index and depressive symptoms. Appetite 52, 238-240.
8. Mikolajczyk RT, El Ansari W \& Maxwell AE (2009) Food consumption frequency and perceived stress and depressive symptoms among students in three European countries. Nutr J 8, 31 .

9. Guo X, Park Y, Freedman ND, et al. (2014) Sweetened beverages, coffee, and tea and depression risk among older US adults. PLOS ONE 9, e94715.

10. Knüppel A, Shipley MJ, Llewellyn CH, et al. (2017) Sugar intake from sweet food and beverages, common mental disorder and depression: prospective findings from the Whitehall II study. Sci Rep 7, 6287 .

11. Segui-Gomez M, Fuente Cdl, Vazquez Z, et al. (2006) Cohort profile: The 'Seguimiento Universidad de Navarra' (SUN) study. Int J Epidemiol 35, 1417-1422.

12. De la Fuente-Arrillaga C, Vazquez Ruiz Z, et al. (2010) Reproducibility of an FFQ validated in Spain. Public Health Nutr 3, 1364-1372.

13. Fernandez-Ballart JD, Piñol JL, Zazpe I, et al. (2009) Relative validity of a semi-quantitative food-frequency questionnaire in an elderly Mediterranean population of Spain. Br J Nutr 103, 1808-1816.

14. Moreiras O, Carbajal A \& Cabrera L (2005) Food Composition Tables, 9th ed. Madrid: Piramide.

15. Mataix Verdu J (2003) Spanish Food Composition Tables, 4th ed. Granada: Granada University.

16. Willett WC, Howe GR \& Kushi LH (1997) Adjustment for total energy intake in epidemiologic studies. Am J Clin Nutr 65 , 4 Suppl., 1220S-1228S; discussion 1229S-1231S.

17. Zazpe I, Santiago S, Gea A, et al. (2016) Association between a dietary carbohydrate index and cardiovascular disease in the SUN (Seguimiento Universidad de Navarra) Project. Nutr Metab Cardiovasc Dis 26, 1048-1056.

18. Sanchez-Villegas A, Schlatter J, Ortuno F, et al. (2008) Validity of a self-reported diagnosis of depression among participants in a cohort study using the Structured Clinical Interview for DSM-IV (SCID-I). BMC Psychiatry 8, 43

19. Martinez-Gonzalez MA, Lopez-Fontana C, Varo JJ, et al. (2005) Validation of the Spanish version of the physical activity questionnaire used in the Nurses' Health Study and the Health Professionals' Follow-up Study. Public Health Nutr 8, 920-927.

20. Bes-Rastrollo M, Perez JR, Sanchez-Villegas A, et al. (2005) Validation of the self-reported weight and body mass index of the participants in a cohort of university graduates. Rev Esp Obes 3, 352-358.

21. Fernandez-Montero A, Beunza JJ, Bes-Rastrollo M, et al. (2011) Validity of self-reported metabolic syndrome components in a cohort study. Gac Sanit 25, 303-307.

22. Alonso A, Beunza JJ, Delgado-Rodríguez M, et al. (2005) Validation of self reported diagnosis of hypertension in a cohort of university graduates in Spain. BMC Public Health 5, 94.

23. Trichopoulou A, Costacou T, Bamia C, et al. (2003) Adherence to a Mediterranean diet and survival in a Greek population. N Engl J Med 348, 2599-2608.

24. Desquilbet L \& Mariotti F (2010) Dose-response analyses using restricted cubic spline functions in public health research. Stat Med 29, 1037-1057.

25. Martinez-Gonzalez MA \& Sanchez-Villegas A (2016) Food patterns and the prevention of depression. Proc Nutr Soc 75, 139-146.

26. Khosravi M, Sotoudeh G, Majdzadeh R, et al. (2015) Healthy and unhealthy dietary patterns are related to depression: a case-control study. Psychiatry Investig 12, 434-442.

27. Akbaraly TN, Brunner EJ, Ferrie JE, et al. (2009) Dietary pattern and depressive symptoms in middle age. $\mathrm{Br} J$ Psychiatry 195, 408-413.

28. Sanchez-Villegas A, Toledo E, de Irala J, et al. (2012) Fast-food and commercial baked goods consumption and the risk of depression. Public Health Nutr 15, 424-432. 
29. Sanchez-Villegas A, Verberne L, De Irala J, et al. (2011) Dietary fat intake and the risk of depression: the SUN Project. PLOS ONE 6, e16268.

30. Markus CR (2007) Effects of carbohydrates on brain tryptophan availability and stress performance. Biol Psychol 76, 83-90.

31. Fernstrom JD \& Wurtman RJ (1972) Brain serotonin content: physiological regulation by plasma neutral amino acids. Science 178, 414-416.

32. Nanri A, Eguchi M, Kuwahara K, et al. (2014) Macronutrient intake and depressive symptoms among Japanese male workers: the Furukawa Nutrition and Health Study. Psychiatry Res 220, 263-268.

33. Aparicio A, Robles F, Lopez-Sobaler AM, et al. (2013) Dietary glycaemic load and odds of depression in a group of institutionalized elderly people without antidepressant treatment. Eur J Nutr 52, 1059-1066.

34. Park JY, You JS \& Chang KJ (2010) Dietary taurine intake, nutrients intake, dietary habits and life stress by depression in Korean female college students: a case-control study. J Biomed Sci 17, Suppl. 1, S40.

35. Stanhope KL, Schwarz JM, Keim NL, et al. (2009) Consuming fructose-sweetened, not glucose-sweetened, beverages increases visceral adiposity and lipids and decreases insulin sensitivity in overweight/obese humans. J Clin Invest 119, 1322-1334.

36. Wang J, Light K, Henderson M, et al. (2014) Consumption of added sugars from liquid but not solid sources predicts impaired glucose homeostasis and insulin resistance among youth at risk of obesity. J Nutr 144, 81-86.

37. Harrell CS, Burgado J, Kelly SD, et al. (2015) High-fructose diet during periadolescent development increases depressivelike behavior and remodels the hypothalamic transcriptome in male rats. Psychoneuroendocrinology 62, 252-264.

38. Aune D, Norat T, Romundstad P, et al. (2013) Whole grain and refined grain consumption and the risk of type 2 diabetes: a systematic review and dose-response meta-analysis of cohort studies. Eur J Epidemiol 28, 845-858.

39. Bhupathiraju SN, Tobias DK, Malik VS, et al. (2014) Glycemic index, glycemic load, and risk of type 2 diabetes: results from 3 large US cohorts and an updated meta-analysis. Am J Clin Nutr 100, 218-232.

40. Mirrahimi A, de Souza RJ, Chiavaroli L, et al. (2012) Associations of glycemic index and load with coronary heart disease events: a systematic review and meta-analysis of prospective cohorts. J Am Heart Assoc 1, e000752.

41. Jenkins DJ, Kendall CW, Augustin LS, et al. (2002) Glycemic index: overview of implications in health and disease. $A m J$ Clin Nutr 76, 266S-273S.
42. Schwingshackl L \& Hoffmann G (2013) Long-term effects of low glycemic index/load vs. high glycemic index/load diets on parameters of obesity and obesity-associated risks: a systematic review and meta-analysis. Nutr Metab Cardiovasc Dis 23, 699-706.

43. Bullo M, Casas R, Portillo MP, et al. (2013) Dietary glycemic index/load and peripheral adipokines and inflammatory markers in elderly subjects at high cardiovascular risk. Nutr Metab Cardiovasc Dis 23, 443-450.

44. Goletzke J, Buyken AE, Joslowski G, et al. (2014) Increased intake of carbohydrates from sources with a higher glycemic index and lower consumption of whole grains during puberty are prospectively associated with higher IL- 6 concentrations in younger adulthood among healthy individuals. J Nutr $\mathbf{1 4 4}$, 1586-1593.

45. Gopinath B, Flood VM, Burlutksy G, et al. (2016) Association between carbohydrate nutrition and prevalence of depressive symptoms in older adults. Br J Nutr 116, 2109-2114.

46. Haghighatdoost F, Azadbakht L, Keshteli AH, et al. (2016) Glycemic index, glycemic load, and common psychological disorders. Am J Clin Nutr 103, 201-209.

47. Breymeyer KL, Lampe JW, McGregor BA, et al. (2016) Subjective mood and energy levels of healthy weight and overweight/obese healthy adults on high-and low-glycemic load experimental diets. Appetite 107, 253-259.

48. Cheatham RA, Roberts SB, Das SK, et al. (2009) Long-term effects of provided low and high glycemic load low energy diets on mood and cognition. Physiol Behav 98, 374-379.

49. Miki T, Eguchi M, Kurotani K, et al. (2016) Dietary fiber intake and depressive symptoms in Japanese employees: The Furukawa Nutrition and Health Study. Nutrition 32, 584-589.

50. Liu X, Cao S \& Zhang X (2015) Modulation of gut microbiotabrain axis by probiotics, prebiotics, and diet. J Agric Food Chem 63, 7885-7895.

51. Sanchez-Villegas A, Henriquez-Sanchez $\mathrm{P}$, Ruiz-Canela M, et al. (2015) A longitudinal analysis of diet quality scores and the risk of incident depression in the SUN Project. BMC Med 13, 197.

52. Etilé F \& Milcent C (2006) Income-related reporting heterogeneity in self-assessed health: evidence from France. Health Econ 15, 965-981.

53. Rothman KJ, Greenland S \& Lash TL (2008) Validity in epidemiologic studies. In Modern Epidemiology, 3rd ed., pp. 128-147 [KJ Rothman, S Greenland and TL Lash, editors]. Philadelphia, PA: Lippincott Williams \& Wilkins.

54. Henriksen RE, Torsheim T \& Thuen F (2014) Loneliness, social integration and consumption of sugar-containing beverages: testing the social baseline theory. PLOS ONE 9, e104421. 